\title{
Zebrafish show their stripes
}

\author{
SCIENTIFIC NAME \\ Danio rerio
}

TAXONOMY

PHYLUM: Chordata

CLASS: Actinopterygii

ORDER: Cypriniformes

FAMILY: Cyprinidae

\section{Physical description}

The zebrafish is a freshwater fish belonging to the minnow family and named for the distinctive pigmented stripes that span the length of its body and broaden to the end of the caudal fin. Males have alternating golden and blue stripes, while females have silver stripes. The fish has a laterally condensed shape with its mouth pointed upwards, and although individuals can grow up to $6.5 \mathrm{~cm}$ in length, their usual length is $4 \mathrm{~cm}$. Zebrafish typically live for $2-4$ years but may have a lifespan of up to 6 years.

\section{Reproduction and life cycle}

In addition to being a common aquarium fish, the zebrafish is also an important vertebrate model organism in research. As early as the 1930s, the zebrafish was being used as a model for studies of development ${ }^{1}$, largely due to its short production time of approximately 3-4 months. Mature females can reproduce every 203 d, laying hundreds of eggs at a time. All major organs appear within $36 \mathrm{~h}$ of fertilization, and hatching takes place 12-36 h later. Another advantage of the zebrafish is the transparency of embryos and larvae, which permits real-time imaging of pathologies as they develop ${ }^{1}$.

\section{Research résumé}

Zebrafish models of a wide variety of human diseases have been generated using genetic methods. One major area of study is the biological processes associated with pigmentation. Recent studies have focused on using the striped pattern of zebrafish as a model for the investigation of mechanisms of animal pigmentation ${ }^{2}$. The zebrafish pigment pattern is generated by a combination of different pigment cell types (or chromatophores), including melanophores, xanophores and iridophores ${ }^{3}$. Molecular genetic studies suggest that the interactions between the different types of pigment cells play a key role in stripe formation ${ }^{4}$. The migratory pathways of the three cell types are differentially regulated, and disruption of any of these pathways can contribute to variations in pigment pattern and colors ${ }^{3}$.

Many genes related to the pigmentation of zebrafish and the formation of skin patterns have been identified and characterized through the generation of mutant strains. These strains can diverge widely in appearance, from the spotted leopard and dali to the thickly striped obelix. Golden is a light-colored mutant that has provided new insight into the genetic basis of pigmentation differences in humans ${ }^{1}$. The golden phenotype in zebrafish, as well as the light-skinned phenotype in humans, is associated with a smaller number, a smaller size and a lower pigment

density of melanosomes (melanin-containing organelles). A single polymorphism in SLC24A5, the human version of the gene affected by the golden mutation, contributes to $25-38 \%$ of the difference in skin color between Africans and Europeans ${ }^{5}$.

The pigment cells of vertebrates are also implicated in human pathologies, including melanoma, one of the most common and most deadly cancers that is caused by the transformation of melanocytes (melanin-producing cells). Melanophores in zebrafish and melanocytes in humans depend on many of the same genes and pathways, and melanomas with characteristics equivalent to those of human melanomas can be induced in zebrafish in order to study the disease ${ }^{6}$.

1. Lieschke, G.J. \& Currie, P.D. Animal models of human disease: zebrafish swim into view. Nat. Rev. Genet. 8, 353-367 (2007).

2. Yamaguchi, M., Yoshimoto, E. \& Kondo, S. Pattern regulation in the stripe of zebrafish suggests an underlying dynamic and autonomous mechanism. Proc. Natl. Acad. Sci. USA 20, 4790-4793 (2007).

3. Kelsh, R.N., Harris, M.L., Colanesi, S. \& Erickson, C.A. Stripes and bellyspots-a review of pigment cell morphogenesis in vertebrates. Semin. Cell Dev. Biol. 20, 90-104 (2008).

4. Inaba, M., Yamanaka, H. \& Kondo, S. Pigment pattern formation by contactdependent depolarization. Science 335, 677 (2012).

5. Cheng, K.C. Skin color in fish and humans: impacts on science and society. Zebrafish 5, 237-242 (2008).

6. Budi, E.H., Patterson, L.B. \& Parichy, D.M. Post-embryonic nerve-associated precursors to adult pigment cells: genetic requirements and dynamics of morphogenesis and differentiation. PLoS Genet. 7, e1002044 (2011). 